\title{
Corrigendum: A new type of tripropeptin with anteiso-branched chain fatty acid from Lysobacter sp. BMK333-48F3
}

Hideki Hashizume, Masayuki Igarashi, Ryuichi Sawa, Hayamitsu Adachi, Yoshio Nishimura and Yuzuru Akamatsu The Journal of Antibiotics (2016) 69, 892-893; doi:10.1038/ja.2016.105

Correction to: The Journal of Antibiotics (2008) 61, 577-582; doi:10.1038/ja.2008.78

The authors of the above article misjudged the stereochemistry of constituent serine residue that was determined by Marfey's method using HPLC, which was the same as the accompanied corrigendum. Our recent analysis using advanced Marfey's method (LC/MS) revealed that the stereochemistry of serine residue was proved to be D-form, as shown below. Resulting chromatograms and the conditions were shown in Supplementary Figure S1.

In addition to the above corrections, we corrected Figure 1 as given below.

The authors would like to apologize for the mistakes.

\section{(A) TPPaiC}
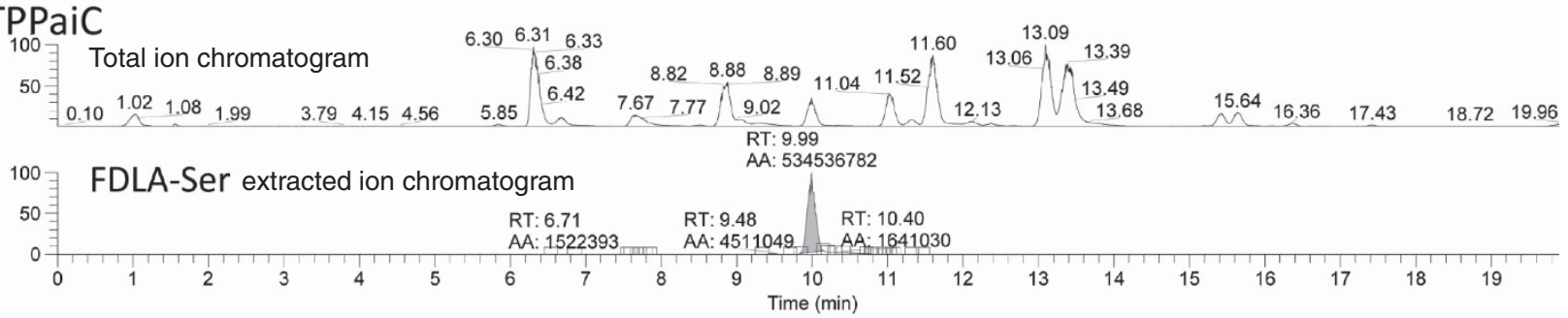

RT: $0.00-20.00$

(B) TPPC

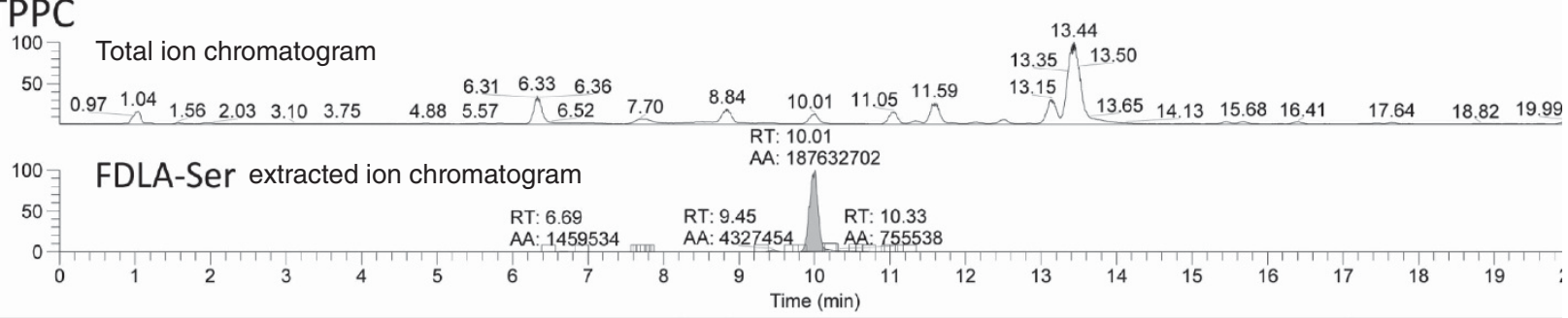

(C)

${ }^{\text {RT }} 0.01{ }^{20.000}$ tic

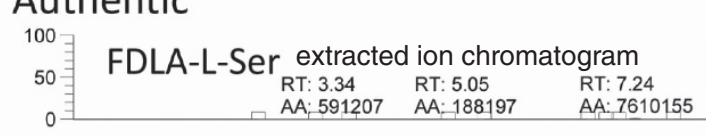

$$
\begin{aligned}
& \text { RT: } 9.49 \\
& \text { AA: } 844134232 \\
& \text { RT: } 8.55 \\
& \begin{array}{c|c}
\text { AA: } 34514 & \text { RT: } 10.05 \\
& \text { AA: } 25163750
\end{array} \\
& \hline \text { RT: } 9.99 \\
& \text { AA: } 800873140
\end{aligned}
$$

Figure S1 Extracted ion chromatograms of L-FDLA derivatives of (A) TPPaiC hydrolysate, (B) TPPC hydrolysate and (C) authentic serines. Acid hydrolysates of tripropeptin aiC (TPPaiC), tripropeptin C (TPPC) and authentic serines were coupled with $N^{\alpha}$-(5-fluoro-2,4-dinitrophenyl)-L-leucinamide (FDLA). Resulting samples were subjected to LC-MS analysis. Total ion chromatograms and extracted ion chromatograms, monitoring at m/z 400.14-400.15 (FDLA coupled serine) were shown. HPLC conditions of Figure S1: Device: LTQ Orbitrap (Thermo Fisher Scientific, Waltham, MA). Column: Shiseido Capcell Pak UG120, $2 \times 150$ mm, $5 \mu \mathrm{m}$. Solvent system: 0-20 min, 20\%A-60\%A linear gradient. A: $\mathrm{CH}_{3} \mathrm{CN}-0.01 \% \mathrm{HCOOH}, \mathrm{B}: 0.01 \% \mathrm{HCOOH}$. Flow rate: $0.3 \mathrm{ml} \mathrm{min}{ }^{-1}$. Temperature: $50{ }^{\circ} \mathrm{C}$. A full color version of this figure is available at The Journal of Antibiotics online. 

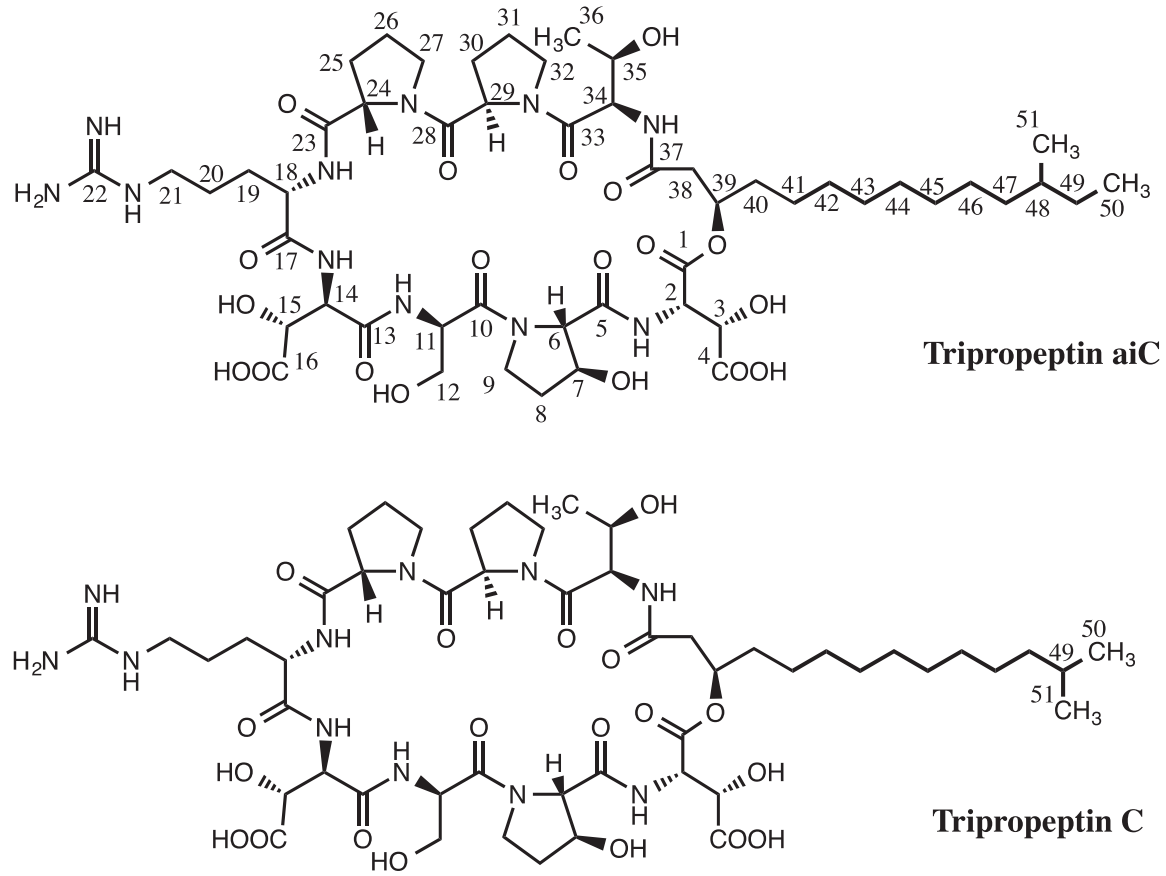

Figure 1 Structure of tripropeptin aiC and tripropeptin C. 